\title{
Framework of consciousness from semblance of activity at functionally LINKed postsynaptic membranes
}

\author{
Kunjumon I. Vadakkan* \\ Division of Neurology, Department of Internal Medicine, University of Manitoba, Winnipeg, MB, Canada
}

\section{Edited by:}

Morten Overgaard, Aarhus University,

Denmark

Reviewed by:

Jessica Saenger, University of

Düsseldorf, Germany

Giorgio Marchetti, Nokia Siemens

Networks - IT, Italy

*Correspondence:

Kunjumon I. Vadakkan, Division of Neurology, Department of Internal Medicine, University of Manitoba, GF532-820 Sherbrook Street,

Winnipeg, MB, Canada R3A1R9.

e-mail: umvadakk@cc.umanitoba.ca
Consciousness is seen as a difficult "binding" problem. Binding, a process where different sensations evoked by an item are associated in the nervous system, can be viewed as a process similar to associative learning. Several reports that consciousness is associated with some form of memory imply that different forms of memories have a common feature contributing to consciousness. Based on a proposed synaptic mechanism capable of explaining different forms of memory, we developed a framework for consciousness. It is based on the formation of semblance of sensory stimulus from (1) synaptic semblances when excitatory postsynaptic potentials arrive at functionally LINKed postsynaptic membranes, and (2) network semblances when these potentials summate to elicit action potential initiating activity in a network of neurons. It is then possible to derive a framework for consciousness as a multi-dimensional semblance. According to this framework, a continuum of semblances formed from background sensory stimuli and oscillating neuronal activities serve to maintain consciousness. Feasibility of this framework to explain various physiological and pathological states of consciousness, its subjective nature and qualia is examined.

Keywords: consciousness, qualia, binding, memory, semblance hypothesis, functional LINKs

\section{INTRODUCTION}

Consciousness is described as a biological and therefore, physical feature of certain higher-level nervous systems of humans and a large number of different types of animals (Searle, 1995). Consciousness is viewed as a hard problem (Chalmers, 1995). Biochemical, electrophysiological, and systems-level studies of the neuronal activities have not allowed us to cross the chasm of consciousness (Blackmore, 2002). However, there is general agreement that brain processes are responsible for consciousness (McGinn, 1989; Searle, 1997) and that finding a perfect match between the contents of the neural and mental states will lead to the discovery of what that process is (McGinn, 1989; Chalmers, 1998). Many investigators have suggested that it is time to approach the problem aggressively with new theories (Gray, 1992) and scientific methods (Farah and Murphy, 2009; Koch, 2009).

Many examinations of the relationship between consciousness and neuronal activities have been made in the past, particularly by examining the continuity in neuronal activity as a possible candidate that can produce a conscious state without any breaks. Oscillatory neuronal networks and their significance in regards to the conscious representation of visual sensory inputs have been studied extensively (Lamme and Spekreijse, 2000; Babiloni et al., 2004; Tallon-Baudry, 2009; Wyart and Tallon-Baudry, 2009). Different frameworks for consciousness based on neuronal activity were made and are being examined (Tononi and Edelman, 1998; Crick and Koch, 2003; Tononi, 2004; Orpwood, 2007; Rolls, 2007; Seth, 2008; He and Raichle, 2009). Even though it is thought that activity patterns and causal interactions between specific groups of neurons could be responsible for consciousness, what constitutes consciousness is not yet known.
While deriving the present framework, theoretical explanations for the following were taken as essential criteria for consciousness. (1) A feasible explanation for qualia is regarded as the gold standard of explanations for consciousness. This is from the view that once a framework of consciousness is developed, then the problem of qualia can be understood better (Crick and Koch, 1998). (2) The discovery of a mechanism that can explain subjective qualities (first-person perspective) of conscious experience is an important parameter of success for deriving a framework of consciousness (Searle, 1992, 1997). (3) A feasible mechanism to accommodate the Zombie phenomenon (Koch and Crick, 2001), where meaningful motor activities can be performed without conscious awareness of them. (4) A mechanism explaining consciousness as a graded, not all-or-none, phenomenon, as evidenced by the varying degrees of loss of consciousness in many clinical settings of acute brain stem injury (Alkire et al., 2008).

A broader degree of philosophical reasoning for the present framework of consciousness was made by integrating information from different fields of brain sciences. The problem was approached by broadening the definition for consciousness by incorporating (1) conscious state, often referred as not required for implicit memory but needed for episodic memory, and (2) consciousness (that is lost during comatose state), which is required for implicit memory. An inductive reasoning approach was used to derive the relationship between consciousness and other brain functions that have a proven direct association with some of the plastic changes at the synapses. Following this, the present work examines the framework's suitability to explain various physiological and pathological conditions associated with conscious states. An abstract of this work was presented at the "Toward a Science of Consciousness" conference, at Tucson, Arizona (Vadakkan, 2010). 


\section{THE CONTEXT}

Studies from various fields of brain sciences have clearly shown that some form of memory is directly associated with consciousness. Short-term memory is thought to be an essential component necessary for consciousness (Ramachandran and Hirstein, 1997). Higher-order consciousness is viewed as dependent on memory for symbols and meanings in the language circuits (Edelman, 1992). With regard to visual consciousness, some form of very short-term transient memory lasting for only a fraction of a second is thought to be essential for consciousness (Crick and Koch, 1998). Using experimental results from a very small number of cases, it has been suggested that semantic memory and general knowledge may contribute to the conscious features of the subjects (Rosenbaum et al., 2007). Conscious machines are thought to require the activation of some memory representations of a device with a certain kind of structure (Minsky, 1991). Since some form of memory is considered to be associated with consciousness, it is reasonable to examine whether a framework for consciousness can be derived from the basic units of memory.

There are two challenging issues that have prevented us from proceeding further. First, the basic mechanism for memory storage is still not known. Second, different forms of memories are thought to have different mechanisms of operation. A feasible approach that can be used at this juncture will be to (1) find a proposal of memory storage that has a common basic unit capable of explaining different types of memories, and (2) find the relationship of the basic unit with the synaptic functions and use the information to develop a framework for consciousness. The framework can then be tested theoretically by examining the relationship between consciousness and memory. Using this method, a framework of consciousness was made from the basic units of the semblance hypothesis of memory (Vadakkan, 2008). Only general principles are explained, leaving aside the specific roles of different sub-regions of the brain such as the reticular activating system, frontal cortex, thalamus, and basal ganglia.

\section{DERIVATION OF THE FRAMEWORK}

The derivation is carried out in two steps. Step one is the derivation of the semblance formation. This is followed by the derivation of the framework of consciousness.

\section{STEP 1: BINDING AND SEMBLANCE FORMATION}

The nervous system is endowed with the property of making associations among different sensory inputs. This can be argued to occur due to the facts that (1) most of the items ("item" means anything that relates to the real world) can induce more than one sensation in the nervous system of an animal having multiple sensory systems, and (2) most animals have nervous systems that can perceive more than one sense. Therefore, while perceiving an item, an animal's nervous system simultaneously receives different sensations from the item and makes a meaningful association between those different sensory inputs (This is similar to learning). The combinatorial activation pattern of different sensory inputs from an item could possibly induce specific signatures in the nervous system for identifying the item at a later stage, when more than one item with similar sensory inputs are present. This has been described as an explicit association, namely "binding" (Rosenblatt,
1961; von der Malsburg, 1981). Out of all the binding problems, the problem of consciousness is considered as the most mystifying (Roskies, 1999).

Most of the natural events in the environment cause changes such that they evoke simultaneous novel inputs to more than one sensory system. For example, the ripening of a banana changes its color from green to yellow. The ripening also leads to changes in the consistency of the banana and thus in the quality of touch. There is a change in taste and the banana becomes edible. The yellowsoft-taste sensory triad creates binding such that either one of these sensations will be sufficient to provide the semblance of the other two sensations. The ability of the yellow color of the banana to provide us with the semblance that it is edible is the consequence of binding. To understand binding, the representations of different sensations from an item and their interaction within the nervous system during binding need to be explored.

The simultaneous arrival of different sensory inputs from one single item can create changes in the nervous system such that at a later point of time, the arrival of one of the sensory inputs will evoke the semblance of arrival of the matching sensations from the same item. This can be viewed as a property of nervous systems with multiple sensory systems. Using reductionistic approaches, we sought to discover synaptic plasticity changes that could evoke appropriate changes during the initial exposure to the item such that at a later occasion, one of the sensory inputs from the item could evoke sensory semblance for the second item. In other words, we examined the elements that are required at the synapses to synthesize a virtual sensation of the remaining sensations from an item when one sensation is activated.

Normally, the arrival of activity from a stimulus at the presynapse (presynaptic terminal) evokes the activation of its postsynapse (postsynaptic terminal or dendritic spine). Activity reaches the presynapse in the form of an action potential and induces an excitatory postsynaptic potential (EPSP) at the postsynapse after neurotransmission. It must be argued that for a sensory semblance to occur, the synapse that is normally activated by one sensation needs to acquire the ability to get activated by the second sensation after the nervous system is exposed simultaneously to both these sensations coming from a single item.

To narrow down the requirements within the synaptic structure, using a reductionistic approach, the following question was asked: "What is the minimum statement that is required, in order to say that one synapse is activated?" Activation of a postsynapse takes place when neurotransmission occurs from its presynaptic terminal. Therefore, the activation of the postsynapse can be taken as the minimum requirement to state that one synapse is activated. Reframing some of the earlier statements, it can be said that coactivation of the postsynapses from the two different sensory inputs from an item induces specific changes that will later allow one of the stimuli alone to evoke the activation of the postsynapse that was originally activated by the second sensory item. In order to achieve this, the postsynapses of the synapses that are activated by the two different sensory inputs from the item should be located in close proximity. Since different sensory inputs converge at many locations within the brain, possible changes that can take place at these locations were examined in hopes of substantiating various properties of consciousness. 
Experimental studies in neuroscience have not yet identified any structural changes between the postsynapses of neighboring synapses at locations where sensory inputs converge. Based on the derivation that was made in the above paragraph, we hypothesize the formation of a functional LINK (capital letters are used in the word LINK to denote its significance) between the postsynapses (Figure 1) at locations where synapses from different sensory inputs converge. Features of functional LINKs are discussed in a separate section.

At a later stage, when one sensory stimulus reaches its synapses and postsynaptic membranes, the functional LINKs can be reactivated such that EPSP can spread from these postsynapses to the neighboring functionally LINKed postsynapses that belong to the second sensory stimulus, causing the latter's activation to spark the semblance of the arrival of that sensation (Figure 2) (mechanisms other than the spread of EPSP through the functional LINKs were also discussed, Vadakkan, 2008). The LINK is called "functional" since (1) LINK formation is a function of the simultaneous activation of different sensory receptors during initial exposures to the item (learning), and (2) reactivation of the LINK is a function of the arrival of EPSP at either one of the postsynapses.

Activation of a postsynapse without corresponding activation of its presynapse will provoke the virtual sensation of activity arriving at the presynapse from the sensory receptors of the second sensation. This is called synaptic semblance. Semblance means that the postsynapse that receives EPSP through the functional LINK experiences the cellular hallucination that it has received an action potential-induced synaptic transmission from its presynapse.

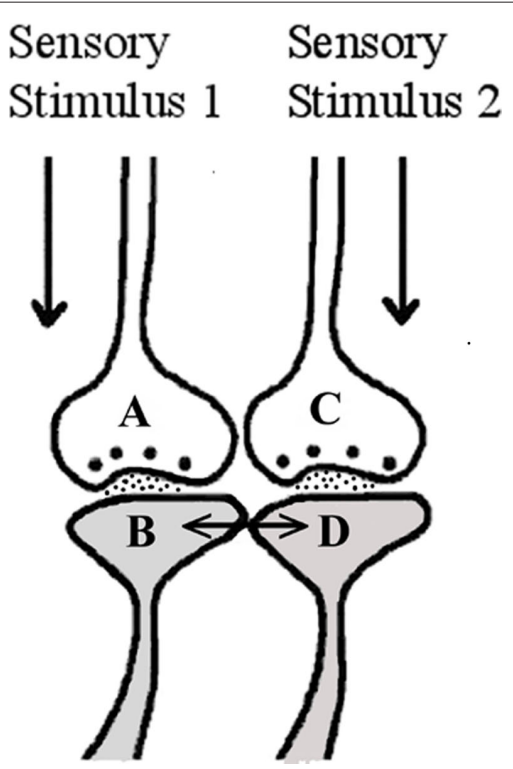

FIGURE 1 | Illustration showing the formation of functional LINK between two postsynaptic membranes $B$ and $D$ during binding. The functional LINK is transient and is a function of the simultaneous arrival of sensory stimuli from an item at the two postsynaptic membranes during binding. This is hypothesized to occur at locations of convergence of sensory inputs. Reactivation of the functional LINK is possible during the arrival of activity at either one of the postsynapses ( $B$ or $D$ ) at a later time (This figure is used after modification [Vadakkan, 2008] with permission from iUniverse publishers, Bloomington, IN, USA).
A large number of different items can induce the activation of the postsynapses B or D and induce synaptic semblance at the synapse on the opposite side of the functional LINK. Thus, even though the functional LINK was formed during binding from a specific item, it can be reactivated by activities of either postsynapses B or D induced by different items. In this context, functional LINKs can be regarded as transferable both during binding and retrieval of bound sensory features representing an item.

Concurrent with the synaptic semblance, the EPSPs reaching the postsynapses through the functional LINKs can travel down to the dendritic tree of the next neuronal order. These EPSPs can undergo spatial and/or temporal summation inducing an action potential at the axon hillock of the neuron. The activity from this neuron propagates in the downstream network of neurons that belong to the second sensory stimulus. Activation of these neurons induces a network semblance (Figure 3) that creates the hallucination of receiving a large subset of the sensory inputs from the second sensory stimulus. From the combined effects of the synaptic and network semblances, the nervous system will be able to receive the virtual sensation of a second sensory stimulus.

In order to translate the semblances to the characteristic features of the virtual sensation of the second stimulus, the following inductive reasoning approach is used. What does semblance means to sensory perception? In other words, how can we derive the sensory quality of a semblance? To answer this question, the sensory equivalent of activity at one presynapse need to be figured out as follows. Let us imagine that synaptic semblance occurs at the postsynapse D (Figure 2). Let presynapse C belongs to neuron $\mathrm{Z}$
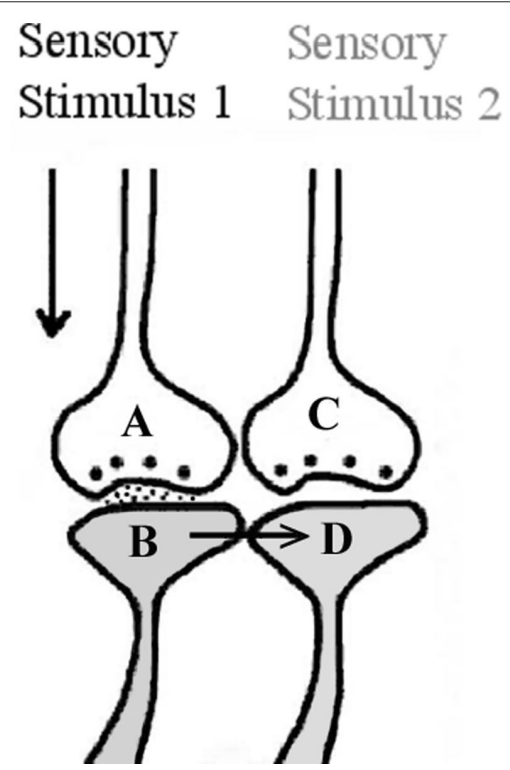

FIGURE 2 |As a result of the binding, when a single sensory stimulus from an item reaches the presynaptic terminal $A$, it depolarizes its postsynaptic membrane $B$, and the resulting EPSP spreads through the functional LINK to postsynaptic membrane $D$. This evokes the cellular hallucination at postsynapse $\mathrm{D}$ of an action potential reaching its presynaptic terminal, C. This is called "synaptic semblance". Note that a functional LINK can be reactivated by EPSP reaching it from either postsynapse $B$ or $D$ (This figure is used after modification (Vadakkan, 2008) with permission from iUniverse publishers, Bloomington, IN, USA). 


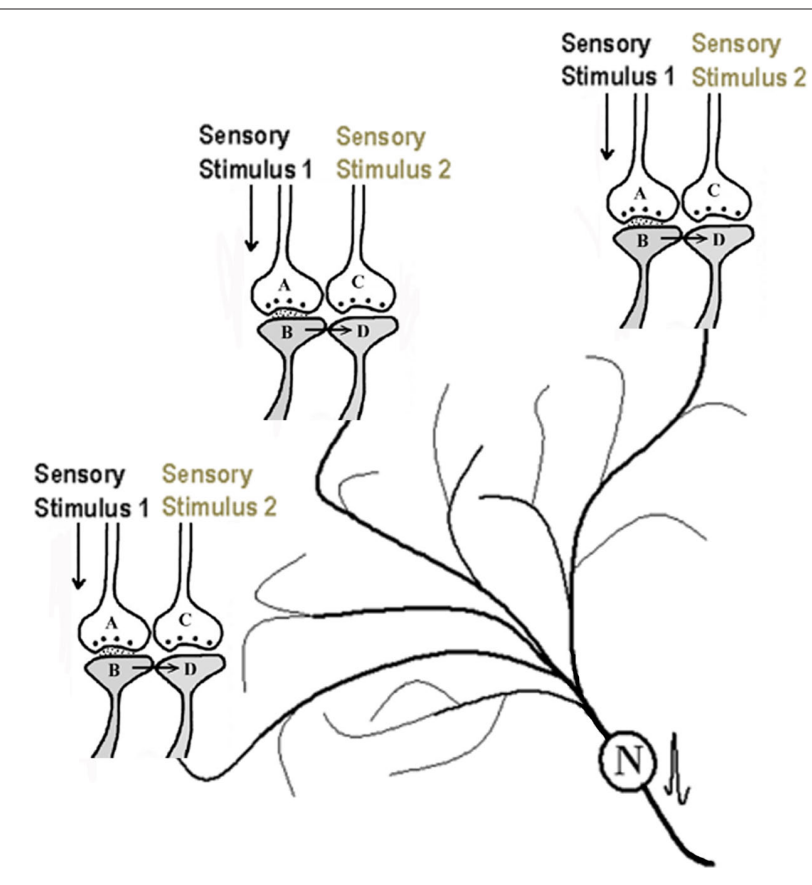

FIGURE 3 | Schematic representation of activation of a partial neuronal network occurring during the arrival of one sensory stimulus from an item after binding (the initial formation of the functional LINK). As explained in Figure 2, synaptic semblances are formed at each of the postsynapses marked D. In addition, the EPSPs from all the " $\mathrm{D}_{n}$ " postsynapses can summate (provided spatial and/or temporal summation of sufficient number of EPSPs is possible) to elicit an action potential at the axon hillock of neuron N. Neuron N belongs to the second sensation. Activating neuron $\mathrm{N}$ will induce the activation of a partial neuronal network that belongs to the second sensory item inducing network semblance.

(Figures 4A,B). Neuron Z, in turn, can only be depolarized by activating a set of axonal terminals belonging to the neurons that synapse to the dendritic spines (postsynapses) on neuron Z's dendritic tree.

The spatial summation of nearly 40 EPSPs or the temporal summation of much less than 40 EPSPs at the soma of neuron $\mathrm{Z}$ can trigger an action potential. Therefore, it is possible that activity from a multitude of possible combinations of any 40 EPSPs (from spatial summation) or permutations of less than 40 EPSPs (from temporal summation) arriving to the neuron Z's nearly $4 \times 10^{4}$ dendritic spines has the ability to trigger the same action potential.

Let us imagine that the dendritic tree of neuron $\mathrm{Z}$ receives activity from a set of neurons $\{Y\}$ through existing synaptic connections that they make with the neuron Z. Similar to this, each neuron synapsing on the neurons in the neuronal set $\{Y\}$ in turn receives synaptic connections from a set of all the possible combinations of neurons upstream from the set of neurons $\{\mathrm{Y}\}$. Let this set of neurons be $\{\mathrm{X}\}$. By moving in this way in a retrograde fashion toward the sensory level, sets of neurons at the preceding neuronal orders can be determined. The last step of this process will determine the set of sensory receptors whose activation can theoretically cause the activation of presynapse C. These sensory receptors are superimposed to obtain the sensory receptor map that represents possible sensory locations which can contribute to the activation of presynapse C (Figure 4D). This sensory receptor map provides the characteristic features of the virtual sensation that is produced by synaptic semblance occurring at the postsynapses.

Since the number of sensory receptors is finite, a large number of sensory inputs from various items can reactivate one functional LINK that was initially formed by binding of sensory stimuli from one item. Therefore, these functional LINKs will be shared for binding of sensory stimuli from different items. This property can be called transferability of the functional LINKs.

The net effect of synaptic and network semblances occurring during the activation of one sensation from an item that results in the virtual sensation of the second sensation in the latter's absence can be called functional semblance. By computing semblances (sensory receptor maps) from all the activated postsynapses, the quality of the functional semblance can be understood. The combination of semblances from different postsynapses at one neuronal order (that are activated almost simultaneously) (Figure 4C) and the permutation of these combination products occurring at the consecutive neuronal orders in a temporal fashion will result in a multi-dimensional net semblance (Figure 4D). The configuration of this net semblance is a function of the complexity of the nervous system and will depend on (1) the genetic make-up of the animal that determines the structural LINKs (structural LINKs can be explained as LINKs between the postsynaptic membranes that are genetically determined and are responsible for innate behaviors), number of neurons, orders of neurons, neuronal organization and convergence pattern of sensory inputs, and (2) functional LINKs acquired during life from previous binding events.

\section{STEP 2: DERIVATION OF THE FRAMEWORK FOR CONSCIOUSNESS}

Continuous formation of semblances is required for the formation of uninterrupted functional semblance for consciousness. The main inputs that provide a continuum of neuronal activities in the nervous system include:

(1) Background sensory inputs. Since an animal cannot possibly shut down all sensory inputs, its nervous system continues to receive background sensory stimuli from the environment. As a result, many sensory receptors are activated. This will induce many synaptic and network semblances contributing to the formation of C-semblance. C-semblance and consciousness are used interchangeably depending on the context.

(2) Neuronal oscillations. The cortex and hippocampus undergo oscillating neuronal activities both during wake-state and sleep. The sets of neurons that are oscillating are unlikely to get activated in the same order during any specific binding events or retrieval of bound sensations. The oscillating population of neurons will induce semblances by reactivating the functional LINKs at their synapses. Since neuronal oscillation occurs in a population of neurons that are highly non-selective for retrieval of any specific sensory item, the synaptic and network semblances induced can be viewed as contributing to C-semblance. 


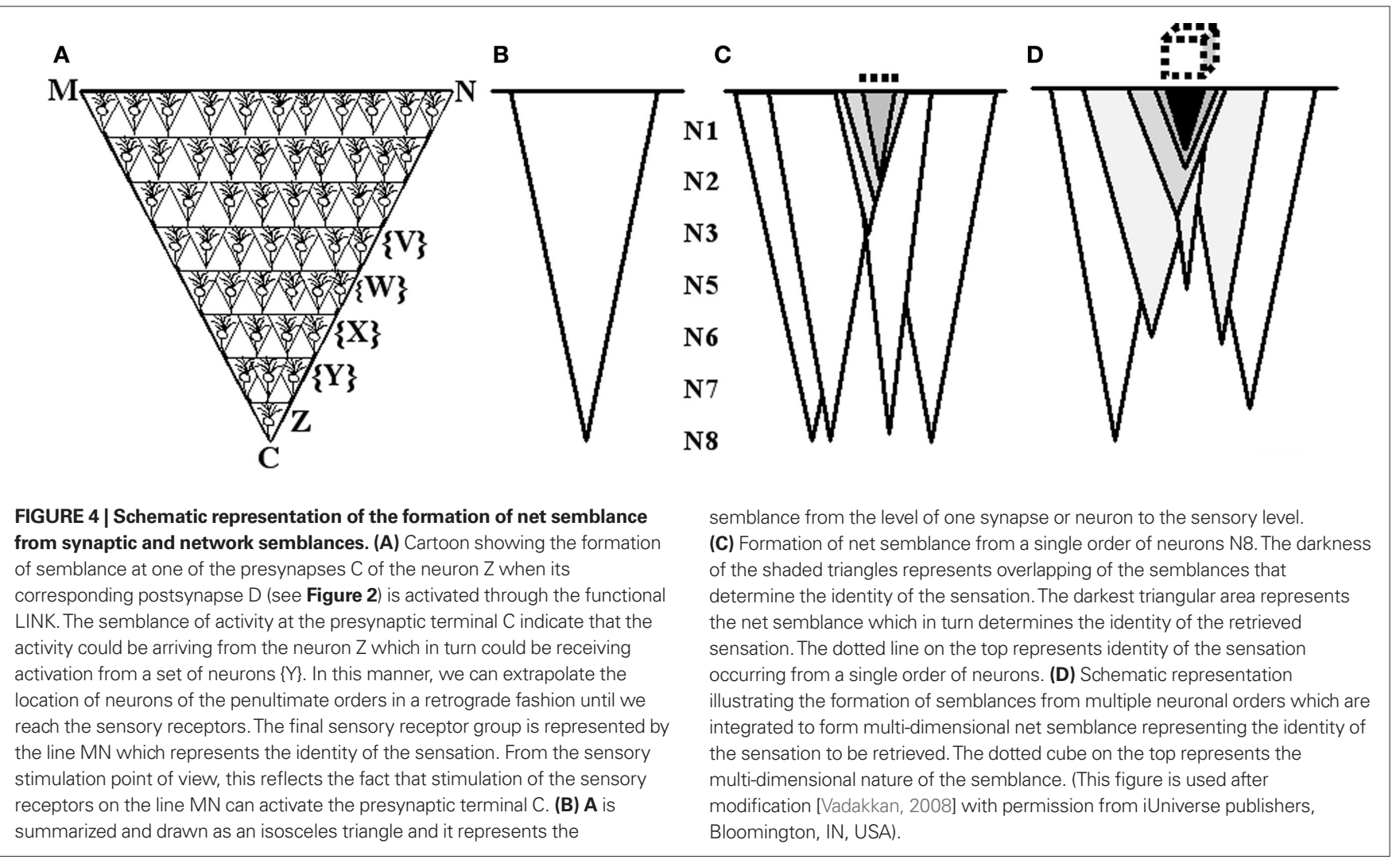

Net functional semblance resulting from the background sensory inputs and neuronal oscillations can be viewed as primary semblance, namely C-semblance, intrinsic to the net semblance formation at a given period in an animal's development. This $\mathrm{C}$-semblance is the basis for consciousness.

$\mathrm{C}$-semblance is a function of the reactivated structural LINKs (which are a function of the complexity of the neuronal architecture of a given species) and functional LINKs (which in turn, are a function of the binding events that occurred prior to the time point when consciousness is tested) during background neuronal activity. The optimum possible C-semblance that can be elicited is considered species-specific. Variations in the individual features of the $\mathrm{C}$-semblance can occur depending on (1) the number of functional LINKs formed during life, and (2) the number of functional LINKs reactivated by the background neuronal activities.

\section{Dimensions of $C$-semblance}

The net $\mathrm{C}$-semblance is formed from the integration of all the synaptic and network semblances occurring at different orders of neurons. Due to the normal synaptic delay, semblances occur at different neuronal orders at different time points, requiring time as one of the dimensions for integration. Oscillating nature of the neuronal network is also likely to add new dimensions based on the phase of the oscillation. The final integration product is a multi-dimensional semblance that leads to the virtual sensation of some non-specific sensory stimuli, which is called C-semblance (Figure 5).

\section{Dynamics of the formation of $C$-semblance}

The contribution of synaptic and network semblances from different regions of the brain that contribute to the $\mathrm{C}$-semblance may vary. Studying isolated lesions of different parts of the brain can help us understand these regional contributions. Brain lesions occurring at different brain locations lead to different levels of unconscious states (Bernat, 2009). Moderate-sized lesions of the cortex are unlikely to affect consciousness as evidenced by reports of lack of changes in conscious states even after the surgical removal of large portions of cortex (Austin and Grant, 1958). It is possible that semblance formation has uniform distribution in the cortex and that there are an excess of functional LINKs in the cortex than that are required to contribute to the formation of C-semblance.

Abrupt loss of consciousness at a critical concentration of an anesthetic agent suggests that the integrated mechanism underlying consciousness collapse non-linearly (Alkire et al., 2008). From this, it may be interpreted that the opposite is possible during the formation of C-semblance. That is, the conscious state is built from its elements as a non-linear exponential function. This means that most of the time, there are more semblances than what is required for $\mathrm{C}$-semblance. This may explain why consciousness is not affected by the surgical removal of a portion of the cortex (Austin and Grant, 1958). On the other hand, changes that can affect the functional LINKs globally, for example, metabolic, electrolyte and toxic effects, may affect C-semblance quickly.

Synchronization of many neuronal activities required for oscillatory network may be useful in maintaining C-semblance. Firstly, it may contribute to a continuum of neuronal activities, without 


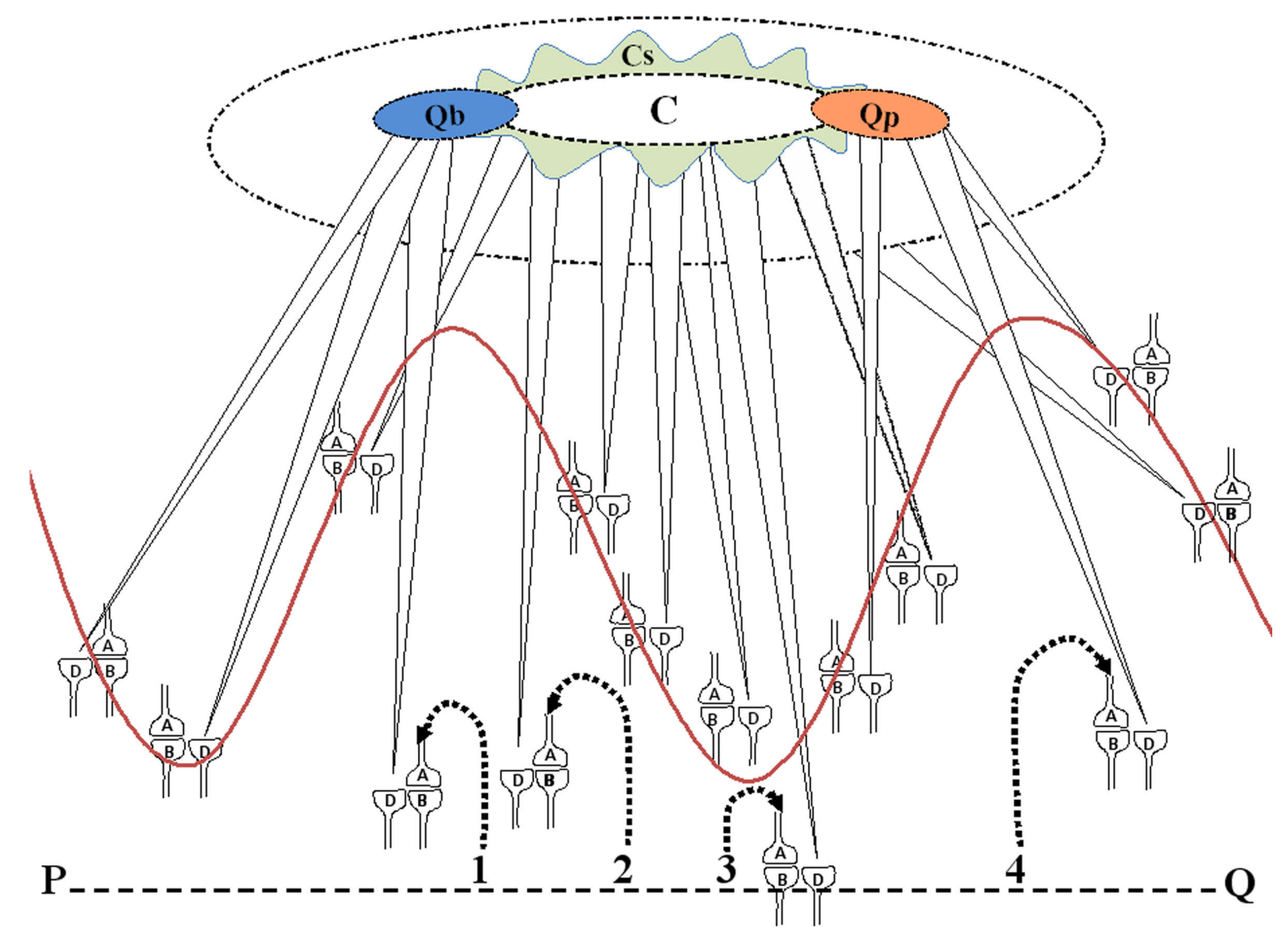

FIGURE 5 | Cartoon showing the formation of primary semblance for consciousness (C-semblance) from the semblances at the LINKed postsynapses as a result of the neuronal oscillations (represented by the wave form) and background sensory inputs (representative inputs are marked as $\mathbf{2}$ and $\mathbf{3}$ from the line PQ which represents the environment). Note the elliptical-shaped net functional semblance $C$ representing consciousness. Around the elliptical-shaped C-semblance, is a wave-shaped extension that represents the subjective variations of $\mathrm{C}$-semblance among the members of a species. Note the center-surround configuration of the

C-semblance. The center denotes the species-specific features and the surround represents the subjective elements of the C-semblance. Formation of secondary semblances for qualia of blueness of the blue (Qb-semblance) and sensation of pain (Op-semblance) are shown. For simplicity, synapses are drawn intersecting the wave form instead of the neuronal soma. The synaptic structures and their labeling are from Figure 2. Ob and $\mathrm{Op}$ semblances depend on the C-semblance and are shown by their overlap. Semblances from the sensory inputs from the items in the environment (represented by sensory inputs marked 1 and 4 from the line $\mathrm{PQ}$ ) provide qualia for the evoked sensation. C, C-semblance (consciousness semblance); Cs, subjective consciousness semblance. any breaks, required for maintaining consciousness. In conditions where the synchrony is broken, it may affect C-semblance. An example of such a condition is absence seizure where conscious states are affected transiently only for a few seconds. Secondly, the synchronized network activity acts as a baseline upon which environmental sensory inputs can induce semblances for memories of associated sensations from different items in the environment. In these respects, synchronization of the neuronal activities may be considered as a binding factor.

\section{Determinants of $\mathrm{C}$-semblance}

Species specificity. Every individual within a species is different in the specific number of neurons and fine details of connectivity patterns within its nervous system. However, all members of a species have a general organization that (1) provides similar distribution of structural LINKs at similar locations, and (2) provides opportunities for the formation of almost similar functional LINKs during life. Individuals of a species living in a given environment will associatively bind (learn) the sensory qualities of almost all the items and this is likely to lead to the formation of almost identical functional LINKs within their nervous systems. The evolving similarities in the structural and functional LINKs can lead to similar C-semblances. Depending on the variations in binding that taking place in each individual, the nervous system develops different number and locations of functional LINKs. These differences are discussed under subjective qualities of C-semblance.

Age of the nervous system. Since functional LINKs are likely absent in a newborn animal, C-semblance will depend on the genetically determined structural LINKs between certain postsynapses, called $(\mathrm{S} \rightarrow \mathrm{F}$ ) LINKs. As the number of binding events an animal undergoes increases, the number of functional LINKs will increase. Since C-semblance in newborn and adult animals varies due to the differences in the number of functional LINKs, the nature of 
consciousness may also vary. Since the conscious state is built from semblances in a non-linear exponential function (discussed in the previous section), the net semblance values after the curve becomes an asymptote indicates that the $\mathrm{C}$-semblances of all members of a species with the same age and environmental exposure are nearly the same.

Interactions of the sensory systems. Injuries of the sensory pathways (Guberman and Stuss, 1983; Steinke et al., 1992) can disrupt consciousness. Acute injuries that damage the brain stem may cause sensory deficits that can evoke an unconscious state (Parvizi and Damasio, 2003). Closure of the eyes induces changes in the electroencephalogram (EEG) wave forms due to changes in the oscillatory pattern of the cortical neurons. This is capable of initiating further changes in the configuration of the wake-state $\mathrm{C}$-semblance. As the sleep cycle progresses, the EEG waveforms keep changing indicating concurrent changes in the configuration of the C-semblance eventually leading to various stages of unconscious stages of sleep.

\section{C-SEMBLANCE AND ITS RELATION TO OTHER SEMBLANCES}

The primary C-semblance is viewed as resulting from the semblances formed from background neuronal activity. In addition, there are many semblances that are formed in response to specific internal or external sensory inputs. Classifying them may provide a better understanding of different types of semblances and their similarities and differences in operation. Secondary semblances are evoked by specific sensory stimuli that reactivate specific functional LINKs. There are two types of secondary semblances: (1) innate semblances that are modified through developmental stages. Examples include those responsible for sight, sound, smell, touch, taste, and pain, (2) semblances formed through the functional LINKs and were acquired through binding. Examples include memory and path finding.

Tertiary semblances can occur when a novel sensory stimulus is presented to a nervous system that has already completed many binding events in the past. The nervous system uses combinations, permutations, and transferability properties to induce net semblances as a response to this novel cue stimulus. Depending on the number of binding events carried out in the past, the nervous system can produce peaks of net semblances that provide novel response patterns. They can interact with external evidence that allows sensory data to veto or support internal constructs which is regarded as a requirement of a system with cognitive abilities (Abbott, 2008). Both secondary and tertiary semblances are likely to depend on the stability of the conformation of the primary C-semblance. The details of their interaction will need further exploration.

\section{SUBJECTIVE QUALITIES OF C-SEMBLANCE}

According to philosophical idealism, things that we perceive around us are the subjective creation of our minds and we have no possibility of knowing what the real world is like (Kant, 1781). Idealism also denies that what we perceive is part of the physical world (Behrendt, 2010). It is viewed that we have no real access to the objective world since we are observing it through our subjective conscious experience (Solomon, 1988). There is an undifferentiated world which exists independently of the observer and differentia- tion takes place at the level of the subject and only exists for the subject (Schopenhauer, 1844). In this context, there are concerns that attempts to associate consciousness to physical properties of the nervous system will lead to negligence of the subjective qualities of the conscious experience (Searle, 1992, 1997). Therefore, a framework for consciousness is expected to incorporate features that can explain these subjective qualities.

C-semblances are expected to be broadly similar among the age-matched members of a species with normal brain functions living in similar environmental conditions. With regard to its fine details, C-semblance is likely to be specific to individual nervous systems at any specific time point. As discussed in the earlier sections, C-semblance is the net effect of the semblances evoked by background neuronal activities. These background neuronal activities can be either induced by or promote reactivation of the functional LINKs formed as a result of specific sensory associations in the nervous system during life. Therefore, depending on the specific functional LINKs present in an individual nervous system, there will be subjective variations in the C-semblance (Figure 5).

For the purpose of simplicity by making pictorial representations, it may be assumed that the $\mathrm{C}$-semblance has a center-surround conformation with center representing the basic $\mathrm{C}$-semblance and the surround representing the subjective variations in consciousness depending on previous binding events (Figure 5). Since semblances are formed only through previously formed functional and structural LINKs, sensing the objective world will inevitably include semblances occurring through the functional LINKs formed during previous binding occurred in an individual.

Another major feature of the subjective aspect of consciousness is the awareness of "self," which is explained as the experiential sense of being a vital and self-coinciding subject of experience or first-person perspective on the world (Sass and Parnas, 2003). The present framework provides provisions to explain how this sensation can arise. Associative binding takes place between our physical body and our actions. Thus, associative binding occurs between the owner of actions and the actions themselves. From this it can be seen that actions, from an individual, specific to the individual form semblance for the owner that provides the sensation of self. This feature of "self" depends on the functional LINKs formed from previous binding events.

\section{QUALIA}

It is expected that a framework for consciousness should be able to support different functional features of qualia (Dennett, 1988; Ramachandran and Hirstein, 1997; Ramachandran and Hubbard, 2001). The perception of the blueness of blue, the painfulness of pain and a certain sound as noise, requires the operation of a mechanism beyond arrival of sensory inputs from the sensory endings to the higher neuronal orders. This is one of the reasons for the difficulty in formulating a mechanism for consciousness (Chalmers, 1995; Shear, 1997; Crick and Koch, 1998).

Based on the present hypothesis qualia can be viewed as secondary semblances namely, qualia semblances (Q-semblances) which are innate to a species, and modified through developmental stages. For example, the color semblance (Qc-semblance) can be viewed as the net effect of all the semblances evoked at different neuronal orders initiated by the activation of color-specific cones. Since 
qualia can only be appreciated in a conscious state, Q-semblances will be dependent on the specific configuration of the primary C-semblance. Similar to the C-semblance, Q-semblances are also multi-dimensional in character and are formed by the combination and permutation of semblances. Q-semblances are dynamic in nature and are a function of reactivation of the LINKs by different sensory stimuli.

It is likely that in the absence of C-semblance, Q-semblances cannot exist. On the contrary, one can imagine conditions having $\mathrm{C}$-semblance, without having certain types of Q-semblances. Even though these Q-semblances are likely to share majority of the semblances from which they were formed with that of the C-semblance, those semblances may not be obligatory for the C-semblance formation. When it comes to the qualia of the consciousness themselves, it is possible that their semblances overlap to a great extent with that of the C-semblance.

\section{LEVELS OF CONSCIOUS AND UNCONSCIOUS STATES}

Awareness, attention, and the ability to respond to sensory stimuli are used to estimate the level of consciousness. Based on the defects in these domains, various conscious states can exist. According to the present framework, changes in the conformation of the multi-dimensional C-semblance can explain these different states. For example, C-semblance sufficient to remain conscious, but not being conscious of a specific motor activity leading to a zombie state (Chalmers, 1996; Koch and Crick, 2001) is possible. During occasions of specific conformational states of C-semblance, a cue stimulus can activate a partial neuronal network stimulating a set of motor neurons (Figure 3 ) and lead to the performance of motor actions without the conscious awareness of the action. In a similar way, an inverse zombie-stateproducing anesthesia awareness (Mashour and LaRock, 2008) can be explained.

Anoxic and traumatic brain injuries cause different chronic consciousness disorders (Bernat, 2009). In addition, many metabolic disorders and medical conditions produce varying levels of consciousness states. Based on the present framework, these disorders can induce different changes ranging from the blockage of sensory stimuli towards the functional LINKs to inhibition of reactivation of the functional LINKs, resulting in lack of formation of appropriate conformation of the C-semblance.

Conscious states are considered separate from attention (Merikle, 1992; Lamme, 2004; Koch and Tsuchiya, 2007). Similar to this, it was reported that awareness is not a prerequisite for learning (Clark and Squire, 1998). While a conscious state is viewed as a multidimensional C-semblance, attention may be viewed as a process required for efficient functional LINK formation for associative learning/binding.

\section{SUPPORTING EVIDENCE}

Any account of the neurobiological processes contributing to conscious perception has to be compatible with various known relationships between different brain functions. If semblances from postsynaptic activation through the functional LINKs are responsible for consciousness, then by disturbing the factors that maintain the functional LINKs, consciousness can be disturbed. This can be used as a test to examine the framework for consciousness in the future. Here, the present framework is examined using currently available data from various physiological and pathological conditions of consciousness.

\section{Anesthesia}

Anesthetic agents cause the inactivation of a complex brain region in the posterior parietal area (Alkire et al., 2008). At low doses of anesthetics, the sustained firing of neurons during normal aroused brain states changes to a bi-stable burst-pause pattern (Llinas and Steriade, 2006) and oscillates between a depolarized up-state and a hyperpolarized down-state (Steriade et al., 2001). The latter is characterized by complete stoppage of synaptic activity for $0.1 \mathrm{~s}$ or more. At higher concentrations of anesthetics, the downstate becomes more prolonged, inducing prolonged stoppage of synaptic activity.

It is possible that in the absence of synaptic activity, functional LINKs stop functioning, leading to a lack of formation of semblances, resulting in unconsciousness. Since time is one of the dimensions that determine the conformation of the C-semblance (by contributing to the permutation of semblances from different neuronal orders that occur at different time points due to synaptic delay) any break in the continuity of activity in the neuronal orders will lead to disturbance in consciousness.

\section{Seizure disorders}

It is known that during seizure activity, a large portion of the cortico-thalamic complex is engaged in strong hyper-synchronous stereotypic activity (Hudetz and Imas, 2007; Kroeger and Amzica, 2007). Since a very large number of non-specific neurons fire during a seizure activity, the semblances occurring at the postsynapses will add up to form a very large non-specific net semblance that changes the conformation of C-semblance. This supports the idea that $\mathrm{C}$-semblance requires a specific conformation (or at least a range of feasible conformations) for consciousness.

\section{Sleep}

During sleep, there is breakdown of cortical effective connectivity (Massimini et al., 2005). Similar to the bi-stable state seen with anesthetics, the hyperpolarized down-states induced by sleep involve changes in background neuronal oscillations, as evidenced by EEG wave form changes (Sanchez-Vives and McCormick, 2000). As neuronal oscillatory patterns change, the conformation of net semblance changes and results in loss of consciousness.

\section{Hypoxia and hypoglycemia}

These can lead to a reduction in the levels of consciousness possibly by interfering with the reactivation of functional LINKs required for sufficient semblances for the formation of C-semblances. Consequent to the defects in consciousness, this can lead to defects in secondary and tertiary semblances.

\section{Chronic consciousness disorders and delirium}

The spectrum of clinical conditions of chronic disorders of consciousness range from minimally conscious state to coma (Bernat, 2009). It is possible that with each of these conditions, there is 
a corresponding spectrum of multi-dimensional conformations of C-semblance. Similarly, delirium is a disorder with fluctuating levels of consciousness. Reducing the background sensory stimuli can worsen the symptoms of delirium, resulting in reduced conscious interactions with the external world. This highlights the role of background environmental stimuli in the formation of C-semblance.

\section{Thalamic relay of sensory inputs}

Activities from almost all sensations pass through different nuclei within the thalamus. Since consciousness depends on background sensory inputs, blocking their transmission can lead to changes in consciousness. The reports that thalamic infarcts cause unconsciousness (Guberman and Stuss, 1983; Steinke et al., 1992) support the present framework and explain the contribution of background sensory inputs to consciousness. In addition, during sleep thalamic neurons undergo a prolonged inhibitory rebound after a single burst (Pedroarena and Llinas, 1997), possibly blocking sensory stimuli from reaching the functional LINKs, interfering with the net semblance formation.

\section{Hippocampus and conscious memory}

Lesions of the hippocampus cause selective loss of declarative or explicit or conscious memory (Cohen and Squire, 1980). Damage to other specific areas of the brain impairs unconscious forms of memory (Bechara et al., 1995; Knowlton et al., 1996). It is reported that hippocampal networks may be required for awareness of the memories of learned item (Eichenbaum, 1999). These findings indicate that the convergence of multi-sensory inputs in the hippocampus and the resulting functional LINK formation at the hippocampus and at higher orders of neurons are necessary for specific semblances for explicit or conscious memory.

\section{Unconscious memory and goal pursuits}

Unconscious associative memory can occur without conscious awareness (Chaumon et al., 2008). In one study, re-exposure to an odor during sleep that had been presented as context during prior learning (Rasch et al., 2007), was shown to improve the retention of hippocampus-dependent declarative memories. It is possible that the functional LINKs were reactivated during the exposure and brought about changes similar to that during repetitive learning. Similarly, actions can be initiated unconsciously (Custers and Aarts, 2010) and can be explained based on the activation of motor neurons as a result of the activation of a partial neuronal network (Figure 3).

\section{Color vision}

Qualia of color are expected to occur from a specific multi-dimensional conformation. Therefore, it is reasonable to expect that reducing the number of neuronal orders through which stimuli from color travel, will affect the qualia by reducing semblances occurring at different neuronal orders. This is observed in the following experiment. As the rotating speed of a wheel of colors increases, perceptional abilities of individual colors decreases. The only color that can be perceived at high speed is white. The difficulty in perceiving individual colors at high speeds can be explained as follows.
Color perception is time-dependent and involves more neuronal orders than required for the quale of the color white. Since eyes cannot fixate on a single color on the rotating wheel for sufficient time, the sensory stimuli will not reach a sufficient number of neuronal orders. This will reduce the required number of neuronal orders from which permutation of semblances needs to be computed for the quale of individual colors. This results in the lack of quale for individual colors. At high speeds, overlapping of semblances for multiple colors from the initial orders of neurons will result in net semblance for color white (Qc-wsemblance).

According to the present framework, the net semblance for color vision requires semblances from more neuronal orders than are required for the color white. Patients with cortical achromatopsia are color blind; but they can see the color white. It is likely that in these patients, semblances are not formed at the higher orders of neurons and thus not contributed to the formation of net semblance for individual colors. Based on the present framework, it is possible to find lesions affecting connections to the higher orders or neuronal loss at the higher orders in these patients.

\section{Modulations of conscious experience}

A previous work (Bottini et al., 1995) has shown that the activation of the vestibular system aided conscious tactile perception in an individual who had lost all abilities of tactile perception due to damage of the sensory pathways of touch at areas before its convergence with the fibers from the vestibular system. Normally, vestibular and tactile systems share projections to the putamen, insula, somatosensory cortex II, premotor cortex, and supramarginal gyrus. The study reported that the vestibular system introduced bias in the neural system involved in body representation. The physiological mechanism of this phenomenon is not yet known.

Based on the present framework, many functional LINKs would have formed between the vestibular system and the pathways governing touch sensation at the locations of convergence of these sensations (in the patient mentioned in the above study, at the orders of neurons after the region of brain damage) during life. At a later time, vestibular sensations alone can induce semblances at the LINKed postsynapses for the touch sensation. The perception of touch sensation occurs only in a conscious state.

\section{Binding problem}

The identification of synaptic mechanisms that can explain binding of association of various sensations has been an unsolved issue for many years. From the example of the ripening of a banana, it can be seen that the yellow-soft-taste sensory triad creates associative functional LINKs between the postsynapses such that either one of these sensations will be sufficient to provide the semblance of the other two sensations.

\section{FUNCTIONAL LINKS BETWEEN THE POSTSYNAPTIC MEMBRANES}

Functional LINKs are defined as LINKs that are formed as a function of simultaneous arrival of EPSPs at the postsynapses that are LINKed during binding or reactivated by the arrival of EPSPs at either one of the postsynapses during the retrieval process. These 
are named functional LINKs since their presence is a function of the arrival of EPSPs. The transient nature of this process may explain the difficulty in exploring its nature. The nature of the functional LINKs and its effect on consciousness can be tested at various levels as following.

1. At the synaptic level: It is anticipated that some specific changes should be taking place between the postsynapses during binding that will allow reactivation of the functional LINKs during retrieval of the bound sensations. The following potential mechanisms that can support the time-scales of retrieval of the bound sensations or memories require further examination.

(a) Different locations of the nervous system show increased blood oxygenation level dependent (BOLD) signal-intensities in functional magnetic resonance imaging (fMRI) studies both during learning and memory retrieval (Cohen et al., 1999; LaBar and Cabeza, 2006). It is reasonable to assume that similar oxygen requiring processes also take place during binding. Oxygen is possibly involved not only to provide energy for the biological process, but also directly involved in the formation of the functional LINKs. Since hippocampus and amygdala are brain regions where different sensory inputs converge (Amaral and Witter, 1995; Doron and Ledoux, 1999), it is most likely that these areas develop large number of functional LINKs. A preliminary report suggestive of the presence of an oxygenation-state dependent functional LINKs was previously made (Vadakkan, 2008). Further exploration of the findings may provide more information about the nature of the functional LINKs. Role of oxygen in the formation and reactivation of the functional LINKs can be tested by using uncouplers of oxidative phosphorylation to examine the selective role of oxygen in the functional LINK formation. Artificially introducing changes at the locations of convergence of sensory inputs that can induce formation of functional LINKs in experimental conditions may be used to study their nature.

(b) The potential spaces between the synapses namely the extracellular matrix (ECM) play an important role in keeping the ionic balance required for maintaining the resting membrane potential. Ephaptic changes that can be brought about between the postsynaptic membranes during binding and its subsequent effect on reactivation of either one of the membranes by activation of the other can be carried out by biophysical studies.

(c) Membrane proteins that can sense the arrival of EPSPs in the neighboring postsynaptic membranes during retrieval of the identities of the bound sensations can induce synaptic semblance reminiscent of the arrival of action potentials from the latter's presynaptic terminals. Proteins that can sense the sequence of ionic changes in the common ECM space during the arrival of action potential may provide valuable information. (d) Inductive depolarization of one postsynapse by another is a feasible mechanism that can be tested. Electroconvulsive stimulation (ECS) that alters the level of consciousness during the post-procedure period may act through disturbing the membrane electrical properties.

2. At the neuronal level: Electrophysiological studies can be carried out to examine the effect of functional LINKs at the neuronal level. A study examining cue-associated learning of reward at the thalamo-amygdalar synapses in the lateral amygdala (Tye et al., 2008) had shown an increase in the miniature EPSP (mEPSP) amplitude after the learning event. This can be explained as a function of additional AMPA channel currents through the functionally LINKed postsynapses. The same work also showed learning-induced firing of additional neurons in the lateral amygdale. This can be explained in terms of activation of a partial neuronal network (Figure 3) through the functional LINKs. Since binding can be viewed as an associative learning, the above explanations can be further explored to understand the nature of the functional LINKs used in building the framework.

3. At the systems level: Examination of the effect of functional LINKs on neuronal oscillation can be studied by testing whether blocking the functional LINKs can affect the retrieval of the bound sensations. fMRI experiments to study the role of functional LINKs in achieving binding and its subsequent effect in the retrieval of the identities of the bound sensations from an item can be carried out.

4. At the behavioral level: If different behavioral effects can be explained based on the role of functional LINKs, then their relationship with consciousness can be examined. For example if functional LINKs can be used to explain some of the features of schizophrenia, then the relationship between consciousness and schizophrenia can be studied.

5. At the epistemological level: It is possible to examine the feasibility of the functional LINKs as a mechanism to explain different brain functions. This will allow us to examine the presence of any cross-correlation between these brain functions and consciousness. From similar approaches carried out to study the role of the functional LINKs in memory (Vadakkan, 2008), it was observed that oxygen, glucose, dopamine, and amphetamine likely augment the formation of the functional LINKs; whereas activation of glycine receptors and gamma amino-butyric acid (GABA)-activated chloride channels, electroconvulsive stimulation and anesthetic agents likely block the functional LINKs. The similarities of the effect of these agents on memory and consciousness can be explored further to understand the nature of the functional LINKs.

\section{SIGNIFICANCE OF C-SEMBLANCE}

$\mathrm{C}$-semblance is viewed as the multi-dimensional virtual sensation of a sensory stimulus resulting from background sensory stimuli and oscillatory neuronal activities taking place at various levels during wake-state. Concurrent with the induction of semblances, the EPSPs pass through the functional LINKs to the postsynapses (dendritic spines) of the dendritic tree of the next order of neurons and induce action potentials, provided sufficient spatial and/or 
temporal summation of EPSPs occurs. During these events, the dendritic trees of many neurons will be short of sufficient EPSPs to induce action potentials at their axonal hillocks. In these neurons that are activated by the background sensory stimuli and neuronal oscillations, the summated EPSPs reaching at sub-threshold levels provide important physiological functions.

At this stage where background neuronal activities that continue to evoke C-semblance keeping the animal conscious, a new cue stimulus reaching the nervous system induces additional functional LINKs that add minor increments in EPSPs and can trigger an action potential in the above-mentioned neurons (that belong to the second sensation) that are otherwise activated at sub-threshold levels. Activation of a set of neurons that belong to one sensation from an item will provide network semblance for retrieval of sensory features from other sensations. This enables the nervous system to utilize the property of binding for its functions. Thus, maintenance of $\mathrm{C}$-semblance aids in retrieval of additional sensory features of an item when only one sensation from the item reaches the nervous system. The same mechanism can explain memory retrieval after learning.

\section{DISCUSSION}

The present work has synthesized a feasible framework using information from various fields of brain sciences. This framework is then examined for the feasibility to support most of the functional requirements for maintaining a conscious state during which other brain functions can be carried out. In summary, the net semblance of activity from the sensory receptors occurring through the transient functional LINKs between the postsynapses by the background sensory receptor activations and neuronal oscillations is explained as the basis of consciousness. The factors that affect the functional LINKs should change the conformation of C-semblance and lead to different levels of consciousness modifying other brain functions that are dependent on C-semblance.

There are difficult questions that need to be answered. For example, can the proposed framework explain basic language comprehension? How does one consciously experience the meaning of the word "and"? Even though, a feasible explanation may be given as follows, is it sufficient? Based on the previous associative learning experience, different semblances for the word "and" are evoked. From them, the right one can be chosen, once we provide the nervous system with the context. Now, the items that need to

\section{REFERENCES}

Abbott, L. F. (2008). Theoretical neuroscience rising. Neuron 60, 489-495.

Alkire, M. T., Hudetz, A. G., and Tononi, G. (2008). Consciousness and anesthesia. Science 322, 876-880.

Amaral, D. G., and Witter, M. P. (1995). "Hippocampal formation," in The Rat Nervous System, 2nd Edn, ed. G. Pacinos (San Diego, CA: Academic Press), 443-493.

Austin, G. M., and Grant, F. C. (1958). Physiologic observations following total hemispherectomy in man. Surgery 38, 239-258.

be added and the word "and" elicit another semblance that contributes to the conscious experience of the process. For example, the word "and" in a mathematical context can bring conscious experience of a plus (+) sign. In the context of mixing two different colors, one would consciously experience the word "and" as mixing them together.

Unique conscious experiences in very complex circumstances are often difficult to explain. However, the present framework provides innumerable combinatorial and permutation possibilities that can create a large number of complex multi-dimensional semblances from nearly $10^{15}$ synapses that are located in multiple neuronal orders. It is possible that retrieval of semblances by certain sensations can cause collapse of the multi-dimensional conformation of C-semblance, similar to discontinuity in the graphs of certain calculus functions leading to loss of consciousness.

The present framework has applied new assumptions that binding introduces reversible changes at the level of synapses and during retrieval a virtual sense of the bound sensations is created. The present work explains consciousness using a framework derived from synaptic plasticity changes. Even though the present work has shown several supportive evidences, empirical verification of the functional LINKs needs to be undertaken. It may be possible to carry out experimental approaches to manipulate the functional LINKs in order to study their effects on consciousness. In addition, it may also become possible to translate the framework for experiments in physical systems.

The present framework raises many challenging questions. How many orders of neurons are required for the conscious state? Can machines have consciousness? Is it wrong to assume that a machine that suitably simulates human brain has consciousness (Minsky, 1986)? Computational work may help to estimate the threshold numbers of neuronal orders that can produce the final effective semblance for consciousness. Since consciousness is a property of the internal state of the nervous system, devising methods to express it using proper read-outs may allow us to understand its nature. The present framework should be treated as unproven until it is verified against further supporting evidence.

\section{ACKNOWLEDGMENTS}

I acknowledge the support from the Neurosearch Center in Toronto, Canada. I thank the reviewers for their questioning minds and insight during various stages of the review process.

Behrendt, R. P. (2010). Contribution of hippocampal region CA3 to consciousness and schizophrenic hallucinations. Neurosci. Biobehav. Rev. 34, 1121-1136.

Bernat, J. L. (2009). Chronic consciousness disorders. Annu. Rev. Med. 60, 381-392.

Blackmore, S. (2002). Crossing the chasm of consciousness. Trends Cogn. Sci. 6 , 276-277.

Bottini, G., Paulesu, E., Sterzi, R., Warburton, E., Wise, R. J., Vallar, G., Frackowiak, R. S., and Frith, C. D. (1995). Modulation of conscious experience by peripheral sensory stimuli. Nature 376, 778-781.

Chalmers, D. (1995). Facing up to the problem of consciousness. J. Conscious. Stud. 2, 200-219.

Chalmers, D. (1996). The Conscious Mind: In Search of a Fundamental Theory. Oxford: Oxford University Press.

Chalmers, D. (1998). "On the search for the neural correlate of consciousness," in Toward a Science of Consciousness II: The Second Tucson Discussions and Debates, eds S. Hameroff, A. Kaszniak, and A. Scott (Cambridge: MIT Press), 219-230. 
Chaumon, M., Drouet, V., and TallonBaudry, C. (2008). Unconscious associative memory affects visual processing before $100 \mathrm{~ms}$. J. Vis. 8, $101-10$.

Clark, R. E., and Squire, L. R. (1998). Classical conditioning and brain systems: the role of awareness. Science 280, 77-81.

Cohen, N. J., Ryan, J., Hunt, C., Romine, L., Wszalek, T., and Nash, C. (1999). Hippocampal system and declarative (relational) memory: summarizing the data from functional neuroimaging studies. Hippocampus 9, 83-98.

Cohen, N. J., and Squire, L. R. (1980). Preserved learning and retention of pattern-analyzing skill in amnesia: dissociation of knowing how and knowing that. Science 210, 207-210.

Crick, F., and Koch, C. (1998). Consciousness and neuroscience. Cereb. Cortex 8, 97-107.

Crick, F., and Koch, C. (2003). A framework for consciousness. Nat. Neurosci. 6, 119-126.

Custers, R., and Aarts, H. (2010). The unconscious will: how the pursuit of goals operates outside of conscious awareness. Science 329, 47-50.

Dennett, D. C. (1988). "Quining Qualia," in Consciousness in Modern Science, eds A. Marcel and E. Bisiach (Oxford: Oxford University Press), 121-158.

Doron, N. N., and Ledoux, J. E. (1999). Organization of projections to the lateral amygdala from auditory and visual areas of the thalamus in the rat. J. Comp. Neurol. 412, 383-409.

Edelman, G. M. (1992). Bright Air, Brilliant Fire: On the Matter of the Mind. New York, NY: Basic Books.

Eichenbaum, H. (1999). Conscious awareness, memory and the hippocampus. Nat. Neurosci. 2, 775-776.

Farah, M. J., and Murphy, N. (2009). Neuroscience and the soul. Science 323, 1168.

Gray, J. (1992). Consciousness on the scientific agenda. Nature 358, 277.

Guberman, A., and Stuss, D. (1983). The syndrome of bilateral paramedian thalamic infarction. Neurology 33, 540-546.

He, B. J., and Raichle, M. E. (2009). The fMRI signal, slow cortical potential and consciousness. Trends Cogn. Sci. 13, 302-309.

Hudetz, A. G., and Imas, O. A. (2007). Burst activation of the cerebral cortex by flash stimuli during isoflurane anesthesia in rats. Anesthesiology 107, 983-991.

Kant, I. (1781). Critique of Pure Reason, translation by J. M. D. Meiklejohn (2003). Mineola, NY: Dover.
Knowlton, B. J., Mangels, J. A., and Squire, L. R. (1996). A neostriatal habit learning system in humans. Science 273, 1399-1402.

Koch, C. (2009). Do not underestimate science. Science 325, 392.

Koch, C., and Crick, F. (2001). The zombie within. Nature 411, 893.

Koch, C., and Tsuchiya, N. (2007). Attention and consciousness: two distinct brain processes. Trends Cogn. Sci. 11, 16-22.

Kroeger, D., and Amzica, F. (2007). Hypersensitivity of the anesthesiainduced comatose brain. J. Neurosci. 27, 10597-10607.

LaBar, K. S., and Cabeza, R. (2006). Cognitive neuroscience of emotional memory. Nat. Rev. Neurosci. 7, 54-64.

Lamme, V. A. (2004). Separate neural definitions of visual consciousness and visual attention; a case for phenomenal awareness. Neural. Netw. 17, 861-872.

Lamme, V. A., and Spekreijse, H. (2000). Modulations of primary visual cortex activity representing attentive and conscious scene perception. Front. Biosci. 5, D232-D243.

Llinas, R. R., and Steriade, M. (2006). Bursting of thalamic neurons and states of vigilance. J. Neurophysiol. 95, 3297-3308.

Mashour, G. A., and LaRock, E. (2008). Inverse zombies, anesthesia awareness, and the hard problem of unconsciousness. Conscious. Cogn. 17, 1163-1168.

Massimini, M., Ferrarelli, F., Huber, R., Esser, S. K., Singh, H., and Tononi, G. (2005). Breakdown of cortical effective connectivity during sleep. Science 309, 2228-2232.

McGinn, C. (1989). Can we solve the mind-body problem? Mind 98, 349-366.

Merikle, P.M. (1992). Perception without awareness. Critical issues. Am. Psychol. 47, 792-795.

Minsky, M. (1986). The Society of Mind. New York: Simon and Schuster Inc. Publishers.

Minsky, M. (1991). "Machinery of Consciousness," Proceedings National Research Council of Canada, 75th Anniversary Symposium on Science in Society.

Orpwood, R. (2007). Neurobiological mechanisms underlying qualia. $J$. Integr. Neurosci. 6, 523-540.

Parvizi, J., and Damasio, A. R. (2003). Neuroanatomical correlates of brainstem coma. Brain 126, 1524-1536.

Pedroarena, C., and Llinas, R. (1997). Dendritic calcium conductances generate high-frequency oscillation in thalamocortical neurons. Proc. Natl. Acad. Sci. U.S.A. 94, 724-728.

Ramachandran, V. S., and Hirstein, W. (1997). Three laws of qualia; What neurology tells us about the biological functions of consciousness. $J$. Conscious. Stud. 4, 429-457.

Ramachandran, V.S., and Hubbard, E. M. (2001). Synaesthesia - a window into perception, thought and language. J. Conscious. Stud. 8, 3-34.

Rasch, B., Buchel, C., Gais, S., and Born, J. (2007). Odor cues during slow-wave sleep prompt declarative memory consolidation. Science 315, 1426-1429.

Rolls, E. T. (2007). A computational neuroscience approach to consciousness. Neural. Netw. 20, 962-982.

Rosenbaum, R. S., Stuss, D. T., Levine, B., and Tulving, E. (2007). Theory of mind is independent of episodic memory. Science 318, 1257.

Rosenblatt, F. (1961). Principles of Neurodynamics: Perceptions and the Theory of Brain Mechanisms. Washington, DC: Spartan Books.

Roskies, A. L. (1999). The binding problem. Neuron 24, 111-125.

Sanchez-Vives, M. V., and McCormick, D. A. (2000). Cellular and network mechanisms of rhythmic recurrent activity in neocortex. Nat. Neurosci. 3, 1027-1034.

Sass, L. A., and Parnas, J. (2003). Schizophrenia, consciousness, and the self. Schizophr. Bull. 29, 427-444.

Schopenhauer,A. (1844). The World as Will and Representation, translated by E. F.J. Payne, 2 vols. New York: Dover.

Searle, J. R. (1992). The Rediscovery of the Mind. Cambridge, MA, USA: Bradford Books/MIT Press.

Searle, J. R. (1995). Construction of Social Reality. New York: The Free Press.

Searle, J. R. (1997). The Mystery of Consciousness. London: Granta Books.

Seth, A. K. (2008). Causal networks in simulated neural systems. Cogn. Neurodyn. 2, 49-64.

Shear, J. (1997). Explaining Consciousness: The Hard Problem. Cambridge, MA: MIT Press.

Solomon, R. (1988). Continental Philosophy since 1750: The Rise and Fall of the Self. Oxford: Oxford University Press.

Steinke, W., Sacco, R. L., Mohr, J. P., Foulkes, M. A., Tatemichi, T. K., Wolf, P.A., Price, T. R., and Hier, D. B. (1992) Thalamic stroke. Presentation and prognosis of infarcts and hemorrhages. Arch Neurol 49, 703-710.

Steriade, M., Timofeev, I., and Grenier, F. (2001). Natural waking and sleep states: a view from inside neocortical neurons. J. Neurophysiol. 85 , 1969-1985.

Tallon-Baudry, C. (2009). The roles of gamma-band oscillatory synchrony in human visual cognition. Front. Biosci. 14, 321-332.

Tononi, G. (2004). An information integration theory of consciousness. BMC Neurosci. 5, 42.

Tononi, G., and Edelman, G. M. (1998). Consciousness and complexity. Science 282, 1846-1851.

Tye, K. M., Stuber, G. D., de Ridder, B., Bonci, A., and Janak, P. H. (2008). Rapid strengthening of thalamo-amygdala synapses mediates cue-reward learning. Nature 453, 1253-1257.

Vadakkan, K. I. (2008). Semblance Hypothesis of Memory. Bloomington, USA: iUniverse Publishers.

Vadakkan, K. I. (2010). Framework of Consciousness from the Semblance Hypothesis of Memory. Toward A Science of Consciousness. Tucson: University of Arizona, Abstract P-8.

von der Malsburg, C. (1981). "The correlation theory of brain function. MPI Biophysical Chemistry, Internal Report 81-2. Reprinted in Models of Neural Networks II (1994)," in Models of Neural Networks II, eds E. Domany, J. L. van Hemmen, and K. Schulten (Berlin: Springer), 95-119.

Wyart, V., and Tallon-Baudry, C. (2009). How ongoing fluctuations in human visual cortex predict perceptual awareness: baseline shift versus decision bias. J Neurosci 29, 8715-8725.

Conflict of Interest Statement: The author declares that the research was conducted in the absence of any commercial or financial relationships that could be construed as a potential conflict of interest.

Received: 26 July 2010; accepted: 23 September 2010; published online: 26 October 2010.

Citation: Vadakkan KI (2010) Framework of consciousness from semblance of activity at functionally LINKed postsynaptic membranes. Front. Psychology 1:168. doi: 10.3389/fpsyg.2010.00168

This article was submitted to Frontiers in Consciousness Research, a specialty of Frontiers in Psychology.

Copyright (c) 2010 Vadakkan. This is an open-access article subject to an exclusive license agreement between the authors and the Frontiers Research Foundation, which permits unrestricted use, distribution, and reproduction in any medium, provided the original authors and source are credited. 